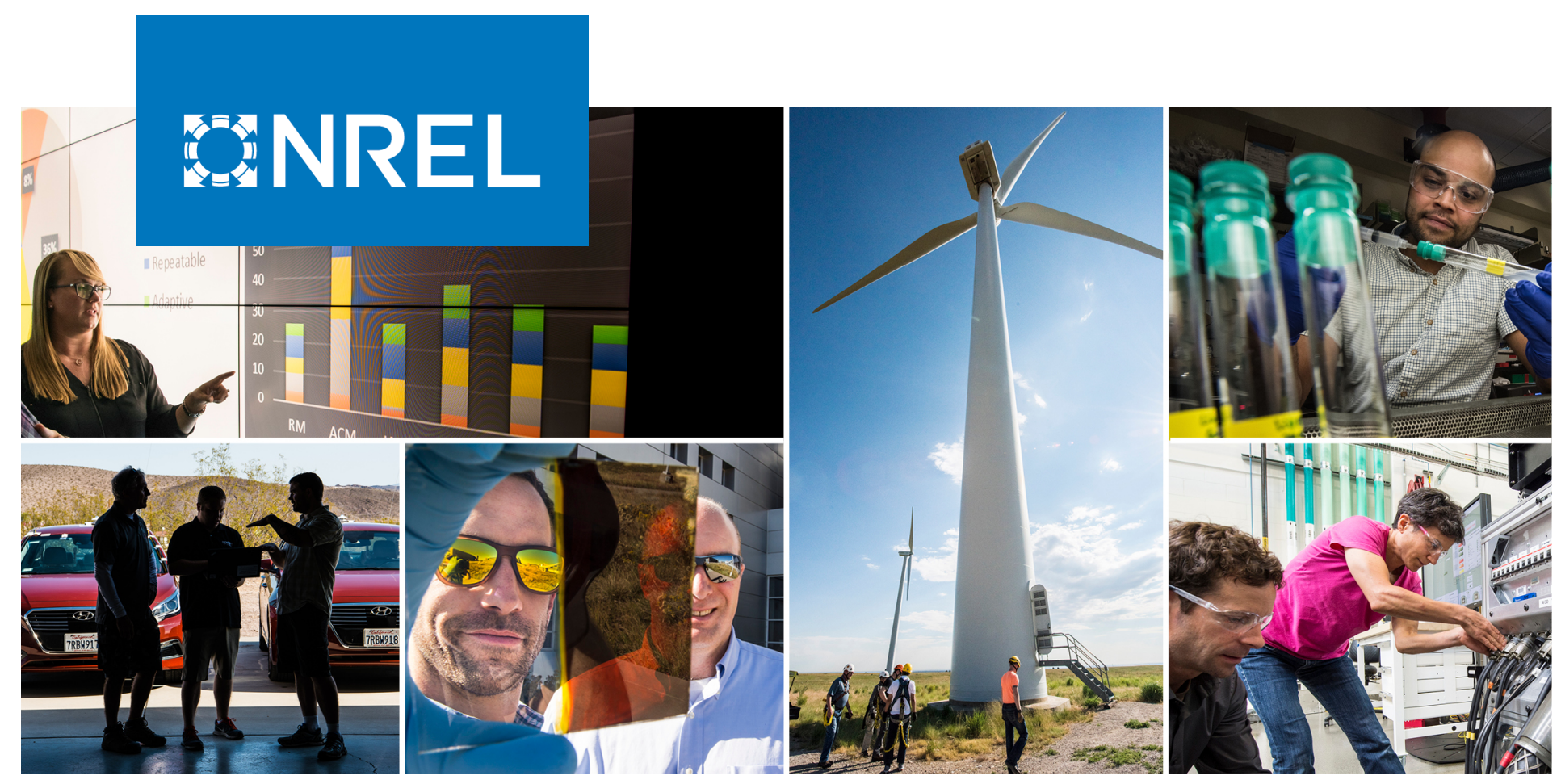

\title{
Decentralized Control of Cascaded H- Bridge Inverters for Medium-Voltage Grid Integration
}

\section{Preprint}

Soham Dutta, ${ }^{1}$ Minghui Lu, ${ }^{1}$ Rahul Mallik, ${ }^{1}$

Branko Majmunovic, ${ }^{2}$ Satyaki Mukherjee, ${ }^{2}$ Gab-Su Seo, ${ }^{3}$ Dragan Maksimovic, ${ }^{2}$ and Brian Johnson ${ }^{1}$

${ }^{1}$ University of Washington

2 University of Colorado, Boulder

${ }^{3}$ National Renewable Energy Laboratory

Presented at 21st IEEE Workshop on Control and Modeling for Power Electronics (IEEE COMPEL 2020)

November 9-12, 2020

NREL is a national laboratory of the U.S. Department of Energy Office of Energy Efficiency \& Renewable Energy

Operated by the Alliance for Sustainable Energy, LLC

This report is available at no cost from the National Renewable Energy Laboratory (NREL) at www.nrel.gov/publications.
Conference Paper

NREL/CP-5D00-78056

December 2020 


\title{
GNREL
}

\section{Decentralized Control of Cascaded H- Bridge Inverters for Medium-Voltage Grid Integration}

\section{Preprint}

\author{
Soham Dutta, ${ }^{1}$ Minghui Lu, ${ }^{1}$ Rahul Mallik, ${ }^{1}$ \\ Branko Majmunovic, ${ }^{2}$ Satyaki Mukherjee, ${ }^{2}$ Gab-Su Seo, ${ }^{3}$ \\ Dragan Maksimovic, ${ }^{2}$ and Brian Johnson ${ }^{1}$ \\ ${ }^{1}$ University of Washington \\ 2 University of Colorado, Boulder \\ ${ }^{3}$ National Renewable Energy Laboratory
}

\section{Suggested Citation}

Dutta, Soham, Minghui Lu, Rahul Mallik, Branko Majmunovic, Satyaki Mukherjee, Gab-Su Seo, Dragan Maksimovic, and Brian Johnson. 2020. Decentralized Control of Cascaded H-Bridge Inverters for Medium-Voltage Grid Integration: Preprint. Golden, CO: National Renewable Energy Laboratory. NREL/CP-5D00-78056.

https://www.nrel.gov/docs/fy210sti/78056.pdf.

(C) 2020 IEEE. Personal use of this material is permitted. Permission from IEEE must be obtained for all other uses, in any current or future media, including reprinting/republishing this material for advertising or promotional purposes, creating new collective works, for resale or redistribution to servers or lists, or reuse of any copyrighted component of this work in other works.

NREL is a national laboratory of the U.S. Department of Energy Office of Energy Efficiency \& Renewable Energy Operated by the Alliance for Sustainable Energy, LLC

This report is available at no cost from the National Renewable Energy Laboratory (NREL) at www.nrel.gov/publications.

Contract No. DE-AC36-08GO28308
Conference Paper

NREL/CP-5D00-78056

December 2020

National Renewable Energy Laboratory 15013 Denver West Parkway Golden, CO 80401

303-275-3000 • www.nrel.gov 


\section{NOTICE}

This work was authored in part by the National Renewable Energy Laboratory, operated by Alliance for Sustainable Energy, LLC, for the U.S. Department of Energy (DOE) under Contract No. DE-AC36-08GO28308. Funding provided by U.S. Department of Energy Office of Energy Efficiency and Renewable Energy Solar Energy Technologies Office. The views expressed herein do not necessarily represent the views of the DOE or the U.S. Government.

This report is available at no cost from the National Renewable Energy Laboratory (NREL) at www.nrel.gov/publications.

U.S. Department of Energy (DOE) reports produced after 1991 and a growing number of pre-1991 documents are available free via www.OSTI.gov.

Cover Photos by Dennis Schroeder: (clockwise, left to right) NREL 51934, NREL 45897, NREL 42160, NREL 45891, NREL 48097, NREL 46526.

NREL prints on paper that contains recycled content. 


\title{
Decentralized Control of Cascaded H-Bridge Inverters for Medium-Voltage Grid Integration
}

\author{
Soham Dutta*, Minghui Lu*, Rahul Mallik*, Branko Majmunovic ${ }^{\dagger}$, Satyaki Mukherjee ${ }^{\dagger}$, Gab-Su Seo ${ }^{\ddagger}$, \\ Dragan Maksimovic ${ }^{\dagger}$, and Brian Johnson* \\ * Department of Electrical and Computer Engineering, University of Washington, Seattle, WA 98195, USA \\ ${ }^{\dagger}$ Department of Electrical, Computer, and Energy Engineering, University of Colorado, Boulder, CO 80309, USA \\ $\ddagger$ Power Systems Engineering Center, National Renewable Energy Laboratory, Golden, CO 80401, USA \\ Corresponding author email: sdutta@uw.edu
}

\begin{abstract}
This paper introduces a fully decentralized control strategy that allows for individualized active and reactive power control of $\mathbf{H}$-bridge inverters connected in series. After formulating a model of the system power flows, we outline a communications-free approach where each inverter can act on independent commands. Inverter-level feedback is accomplished locally via a measurement of current flowing through the stack. A key feature is the lack of any centralized control routines or phase-locked loop. In this framework, the reactive power control loops achieve voltage synchronization among the converters and the grid, and the active power control loops modulate the terminal voltage amplitudes that influence power delivery. Furthermore, pole placement via state feedback is used to ensure stability of voltage synchronization while also enabling tracking of bidirectional power commands. Simulations are presented to validate the proposed approach.
\end{abstract}

\section{INTRODUCTION}

Systems of cascaded inverters appear in a variety of applications, such as solid-state transformers, modular multilevel converters, medium-voltage grid-connected converters, and systems with high-voltage conversion ratios in general. In settings with distinct energy resources or loads behind each dc link, it is desirable to modulate the power transferred from individual $\mathrm{H}$-bridge inverters to achieve objectives such as maximum power point tracking, battery charge control, or load voltage regulation. To avoid single points of failure and obtain a modular structure that streamlines installation, a decentralized solution where each inverter can independently modulate the power processed by it via local measurements is desirable; however, the series-connected nature of such a system exhibits tight coupling that seemingly precludes a decentralized solution. In light of this challenge, established methods generally rely on a centralized controller that processes multiple measurements and modulates every unit in the

Funding was provided in part by the U. S. Department of Energy Office of Energy Efficiency and Renewable Energy Solar Energy Technologies Office grant number DE-EE0008346. This work was authored in part by Alliance for Sustainable Energy, LLC, the manager and operator of the National Renewable Energy Laboratory for the U.S. Department of Energy (DOE) under Contract No. DE-AC36-08GO28308. Brian Johnson was also supported by the Washington Research Foundation. The U.S. Government retains and the publisher, by accepting the article for publication, acknowledges that the U.S. Government retains a nonexclusive, paid-up, irrevocable, worldwide license to publish or reproduce the published form of this work, or allow others to do so, for U.S. Government purposes. The views expressed in the article do not necessarily represent the views of the DOE or the U.S. Government.

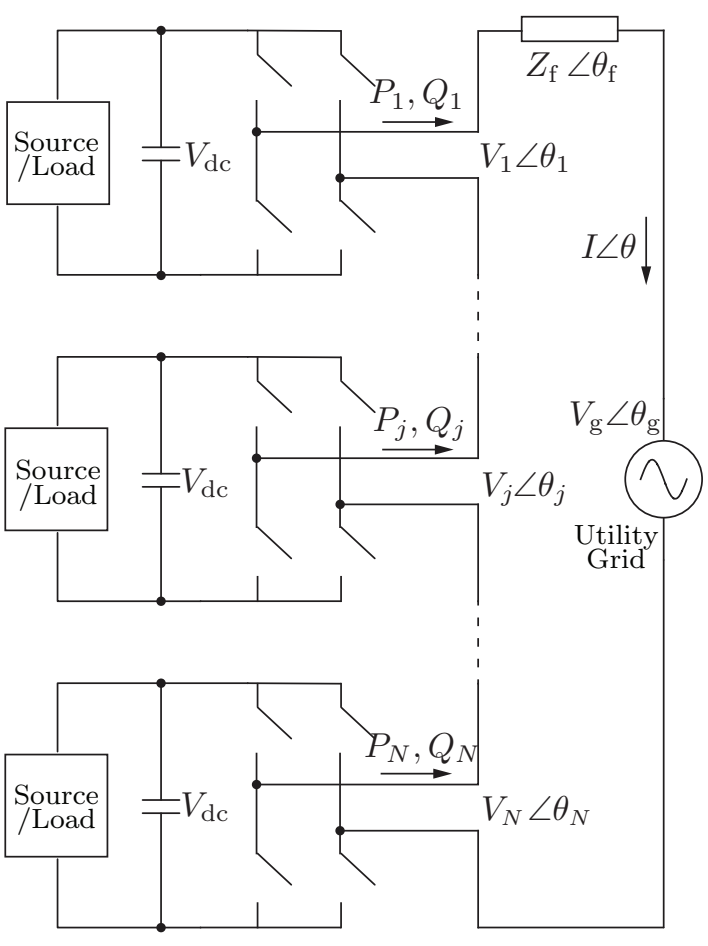

Fig. 1. System of series connected H-bridge inverters.

system. In this paper, we formulate a control strategy that enables a truly decentralized structure without any centralized control and does not contain a phase-locked-loop (PLL). Each inverter in the stack is able to not only synchronize with the grid and other units but also modulate its power independently from others in the system. The proposed controller is presented with generic active and reactive power control loops that can be adapted to a variety of applications.

Before delving into the details of the proposed solution, we will first delineate related prior art. The most straightforward approaches utilize either a centralized controller to dispatch supervisory control commands [1]-[5] or distributed controllers with some sort of low-bandwidth communications for information exchanges [6]-[12] (e.g., grid voltage and angle broadcast, inverter stack voltage amplitude, phase information etc.). The key drawback is that these methods necessitate significant wiring, the controller acts as a single point of 
vulnerability, and implementation on very large numbers of units is impractical even if the required communication is low bandwidth or intermittent. To bypass these issues, recent efforts have been focused on devising decentralized methods where inverters take local control action. In [13], an inverse active power versus power factor droop method is proposed to control active power flow. In [14], a virtual oscillator-based controller is implemented to synchronize the inverter voltages and share the load. However, these approaches are limited to islanded settings and only considers unidirectional power flow.

Decentralized control of grid-connected systems is addressed in [15], [16] where conventional active-power versus frequency droop law is utilized to control active power processed by the inverters. In both of these works, inverter voltage amplitude is held constant at a suitable value dictated by the reactive power delivered and the droop law essentially adjusts the power factor of the inverters to adjust their active power output. The main drawback here is that stability of the active power controller depends on the reactive power processed by the system and in order to obtain a high power factor operation the stability of the system needs to be compromised. Moreover, the control of reactive power is not addressed and only unidirectional power flow is covered.

To overcome these shortcomings, we formulate a novel controller that gives independent control of both active and reactive power processed by each inverter as well as communication-free synchronization. The trade-off between system stability and power factor is eliminated in two ways,a) by altering the network type from an originally inductive network to a resistive network through virtual resistance emulation and, b) by changing the system closed loop pole locations via state feedback to ensure stability during positive active power flow. In the proposed dual-loop setup, the voltage amplitude and angular dynamics are manipulated by active and reactive power control loops, respectively. The resulting system is independent of the number of modules in the stack, which enables flexible plug-and-play operation.

The remainder of this paper is organized as follows: Section II provides an overview of the system modeling and an analysis of the active and reactive power processed by the inverter units with virtual resistance emulation. Section III contains a description of the proposed control method and discussions on design and small-signal stability analysis of the reactive and active power controllers. A modified reactive power control loop incorporating state feedback is also proposed in this section that ensures stable operation during active power delivery to the grid. Section IV presents simulation results to substantiate the proposed control method. Finally, Section V concludes the paper.

\section{Series-Stacked Converter Power Flow ANALYSIS}

The system under consideration in Fig. 1 is composed of $N$ series-connected H-bridge inverters that collectively deliver common current, $I \angle \theta$, into the grid voltage, $V_{\mathrm{g}} \angle \theta_{\mathrm{g}}$, via a network impedance $Z_{\mathrm{f}} \angle \theta_{\mathrm{f}}$. The output voltage phasor, active

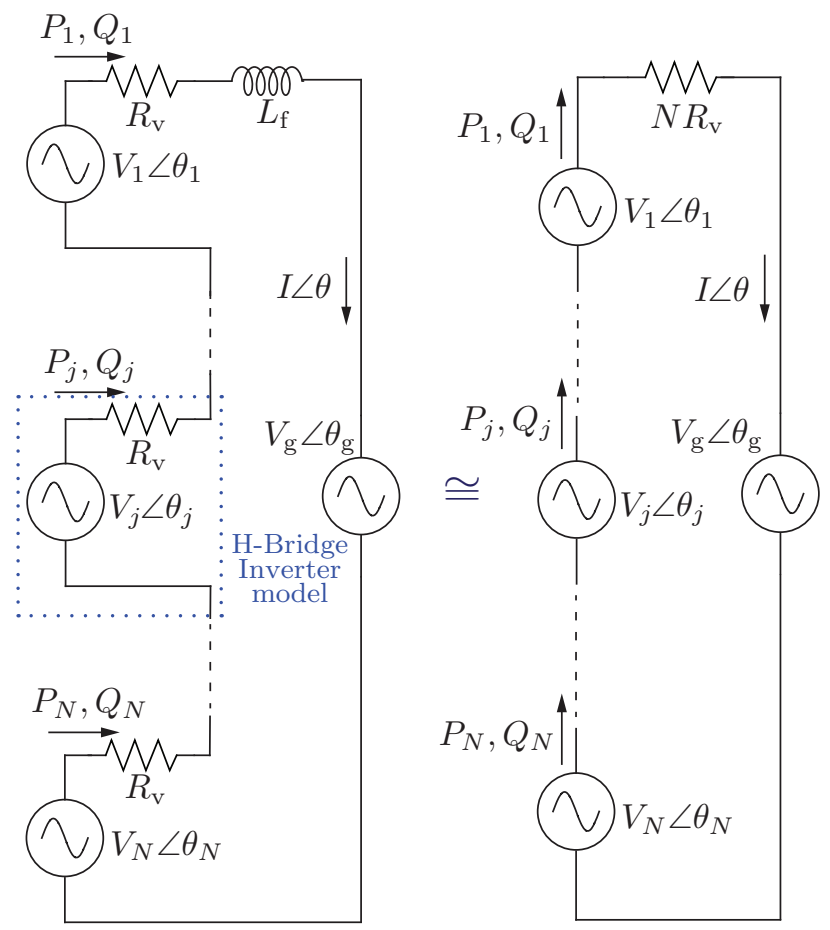

Fig. 2. The output terminals of each H-bridge are modeled as a controllable voltage source behind an emulated resistance $R_{\mathrm{v}}$. The equivalent network on the right is resistive with a net resistance of $N R_{\mathrm{v}}$ as, $N R_{\mathrm{v}} \gg \omega_{\mathrm{O}} L_{\mathrm{f}}$.

power, and reactive power output of the $j^{\text {th }}$ inverter are denoted as $V_{j} \angle \theta_{j}, P_{j}$, and $Q_{j}$, respectively, for $j=1, \cdots, N$, where, $\angle \theta_{j}$ is the angle of the $j^{\text {th }}$ inverter voltage. Accordingly, the active and reactive power output of the $j^{\text {th }}$ inverter can be expressed as

$$
\begin{aligned}
P_{j} & =\sum_{k=1}^{N} \frac{V_{j} V_{k}}{Z_{\mathrm{f}}} \cos \left(\theta_{j k}+\theta_{\mathrm{f}}\right)-\frac{V_{j} V_{\mathrm{g}}}{Z_{\mathrm{f}}} \cos \left(\theta_{j \mathrm{~g}}+\theta_{\mathrm{f}}\right), \\
Q_{j} & =\sum_{k=1}^{N} \frac{V_{j} V_{k}}{Z_{\mathrm{f}}} \sin \left(\theta_{j k}+\theta_{\mathrm{f}}\right)-\frac{V_{j} V_{\mathrm{g}}}{Z_{\mathrm{f}}} \sin \left(\theta_{j \mathrm{~g}}+\theta_{\mathrm{f}}\right),
\end{aligned}
$$

where, $\theta_{j k}$ denotes the angle between the $j^{\text {th }}$ and $k^{\text {th }}$ inverter, and $\theta_{j \mathrm{~g}}$ denotes the angle between the $j^{\text {th }}$ inverter and the grid. Note that (1)-(2) imply cross-coupling of active and reactive power with the voltage amplitudes and angles of each inverter. The properties of $Z_{\mathrm{f}} \angle \theta_{\mathrm{f}}$ dictate the nature of this coupling. In general, $Z_{\mathrm{f}} \angle \theta_{\mathrm{f}}$ arises due to the inverter output filter impedance and the line impedance, which often has a small magnitude and can have inductive as well as significant resistive components that depend on the network type. This prevents complete decoupling of the active and reactive power which necessitates modification of the droop laws to account for the complex nature of $Z_{\mathrm{f}} \angle \theta_{\mathrm{f}}$ [17]. To mitigate this cross-coupling and facilitate a fully de-coupled and decentralized control strategy, we implement a virtual resistor, $R_{\mathrm{v}}$, at each set of terminals via digital control such that $N R_{\mathrm{v}} \gg \omega_{\mathrm{o}} L_{\mathrm{f}}$, where $\omega_{\mathrm{o}}$ is the nominal line frequency (see Fig. 2). This ensures that network impedance is predominantly resistive such that $Z_{\mathrm{f}}=N R_{\mathrm{v}}+\omega_{\mathrm{o}} L_{\mathrm{f}} \approx N R_{\mathrm{v}}$ and 
$\theta_{\mathrm{f}}=\arctan \left(\omega_{\mathrm{o}} L_{\mathrm{f}} /\left(N R_{\mathrm{v}}\right)\right) \approx 0$. Assuming angle differences are sufficiently small in steady state, (1)-(2) can be approximated as:

$$
\begin{aligned}
P_{j} & \approx \sum_{k=1}^{N} \frac{V_{j} V_{k}}{Z_{\mathrm{f}}}-\frac{V_{j} V_{\mathrm{g}}}{Z_{\mathrm{f}}}, \\
Q_{j} & \approx \sum_{k=1}^{N} \frac{V_{j} V_{k}}{Z_{\mathrm{f}}} \theta_{j k}-\frac{V_{j} V_{\mathrm{g}}}{Z_{\mathrm{f}}} \theta_{j \mathrm{~g}} .
\end{aligned}
$$

Note that in (3)-(4), the first summation term captures energy exchanges between inverters, and the second term captures exchanges between the inverters and grid.

\section{A. Steady-State Analysis}

To lay the groundwork for control design and analysis, we first consider a steady-state condition where all inverters are processing equal active power, $P_{\mathrm{o}}$. In this setting, all inverters deliver an arbitrarily small value of reactive power, $Q_{\mathrm{o}}$, and the power factor is $\cos \phi$, which is very close to unity. Hence:

$$
\begin{aligned}
& P_{1 \mathrm{o}}=P_{2 \mathrm{o}}=\cdots=P_{N \mathrm{o}}=P_{\mathrm{o}}, \\
& Q_{1 \mathrm{o}}=Q_{2 \mathrm{o}}=\cdots=Q_{N \mathrm{o}}=Q_{\mathrm{o}}=P_{\mathrm{o}} \tan \phi, \\
& V_{1 \mathrm{o}}=V_{2 \mathrm{o}}=\cdots=V_{N \mathrm{o}}=V_{\mathrm{o}},
\end{aligned}
$$

where (7) follows from (5) and (3). Henceforth, the "o" subscript indicates a steady-state quantity. Since in steady state all the modules process equal reactive power, the angle difference between any two inverter modules is zero, and their output voltages are synchronized (i.e, $\theta_{j k \mathrm{o}}=0 \forall j, k \in\{1, \ldots, N\}$ ). Therefore, from (2), each inverter has the following two solutions for the angle difference with grid,

$$
\theta_{j \mathrm{go}}=: \theta_{\mathrm{o}}=-\arcsin \left(\frac{Z_{\mathrm{f}} Q_{\mathrm{o}}}{V_{\mathrm{o}} V_{\mathrm{g}}}\right), \pi+\arcsin \left(\frac{Z_{\mathrm{f}} Q_{\mathrm{o}}}{V_{\mathrm{o}} V_{\mathrm{g}}}\right) \text {. }
$$

For arbitrarily small values of $Q_{\mathrm{o}}$,

$$
\theta_{\mathrm{o}} \approx 0, \pi
$$

Note here that $\theta_{0} \approx 0$ is the desired equilibrium point as this ensures voltage synchronization of the stack with the grid. After substitution of (5) and (7) into (1), the solution for the steady-state voltage is

$$
V_{\mathrm{o}}=\frac{V_{\mathrm{g}} \cos \theta_{\mathrm{o}} \pm \sqrt{V_{\mathrm{g}}^{2} \cos ^{2} \theta_{\mathrm{o}}+4 N P_{\mathrm{o}} Z_{\mathrm{f}}}}{2 N}=\frac{V_{\mathrm{g}}}{M},
$$

where $M$ is a variable which expresses the steady-state inverter voltage as a fraction of the grid voltage $V_{\mathrm{g}}$. (9)-(10) gives rise to a number of equilibrium points for the steady state power angle $\theta_{\mathrm{o}}$ and inverter voltage amplitude $V_{\mathrm{o}}$ based on $\theta_{\mathrm{o}}$ and sign of $M$. These equilibrium points can be classified into 4 types, - (i) $\theta_{\mathrm{o}} \approx 0$ and $M>0$, (ii) $\theta_{\mathrm{o}} \approx 0$ and $M<0$, (iii) $\theta_{\mathrm{o}} \approx \pi$ and $M>0$, and (iv) $\theta_{\mathrm{o}} \approx \pi$ and $M<0$. Since, the instantaneously generated inverter voltage is, $v_{j}=\sqrt{2} V_{\mathrm{o}} \sin \left(\omega_{\mathrm{o}} t+\theta_{\mathrm{o}}\right)$, cases (i) \& (iv) and cases (ii) \& (iii) gives rise to same inverter voltage. Therefore, it is sufficient to analyze the stability of the steady states given by cases (i) and (ii).

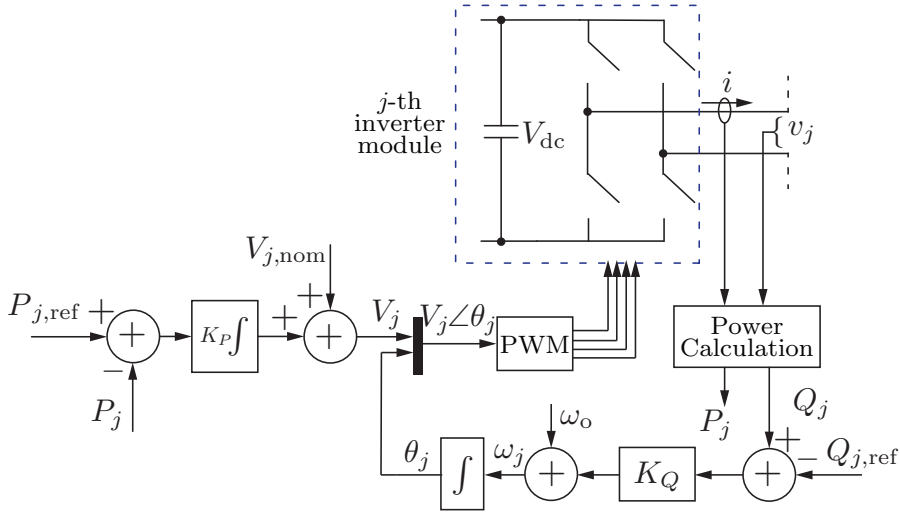

Fig. 3. Control block Diagram for the $j^{\text {th }}$ inverter module.

\section{Proposed Decentralized Control Method}

From (3)-(4), it follows that the reactive and active power outputs are governed by the output voltage angles and amplitudes, respectively. Based on this observation, the underlying principle behind the proposed method is to: i) synchronize the angles of the voltage waveforms with reactive power control, and ii) modulate active power transfer via voltage amplitude control. To achieve these aims, the proposed control law is

$$
\begin{aligned}
& \omega_{j}=\omega_{\mathrm{o}}-K_{Q}\left(Q_{j, \mathrm{ref}}-Q_{j}\right), \\
& V_{j}=V_{j, \text { nom }}+K_{P} \int\left(P_{j, \mathrm{ref}}-P_{j}\right) d t,
\end{aligned}
$$

where $\omega_{j}$ is the $j^{\text {th }}$ inverter frequency; $V_{j, \text { nom }}$ is the nominal voltage amplitude; $K_{Q}$ and $K_{P}$ are the reactive and active power controller gains, respectively; and $Q_{j, \text { ref }}$ and $P_{j \text {,ref }}$ are the reactive and active power references for the $j^{\text {th }}$ inverter module, respectively. The implementation of this control method is shown in Fig. 3.

\section{A. Stability Analysis \& Design of Q-control Loop}

Given small angle perturbations around the steady-state point in (2), the small-signal reactive power output of the $j^{\text {th }}$ inverter is approximated as

$$
\begin{aligned}
\widetilde{Q}_{j} \approx & \frac{V_{j \mathrm{o}}}{Z_{\mathrm{f}}}\left(\sum_{k \neq j}^{N} V_{k o}-V_{\mathrm{g}} \cos \theta_{j \mathrm{go}}\right) \widetilde{\theta}_{j}-\frac{V_{j \mathrm{o}}}{Z_{\mathrm{f}}} \sum_{k \neq j}^{N} V_{k \mathrm{o}} \widetilde{\theta}_{k} \\
& -\frac{V_{\mathrm{g}} \sin \theta_{j \mathrm{go}}}{Z_{\mathrm{f}}} \widetilde{v}_{j} .
\end{aligned}
$$

Using (8), it follows that small values of $Q_{\mathrm{o}}$ imply $\sin \theta_{j \text { go }} \approx$ 0 ; hence, (13) becomes

$$
\widetilde{Q}_{j} \approx \frac{V_{j \mathrm{o}}}{Z_{\mathrm{f}}}\left(\sum_{k \neq j}^{N} V_{k \mathrm{o}}-V_{\mathrm{g}} \cos \theta_{j \mathrm{go}}\right) \widetilde{\theta}_{j}-\frac{V_{j \mathrm{o}}}{Z_{\mathrm{f}}} \sum_{k \neq j}^{N} V_{k \mathrm{o}} \widetilde{\theta}_{k} .
$$

To evaluate the small-signal stability, we formulate a model with the state vector $\widetilde{\theta}=\left[\widetilde{\theta}_{1}, \ldots, \widetilde{\theta}_{N}\right]^{\top}$, output vector $\widetilde{Q}=\left[\widetilde{Q}_{1}, \ldots, \widetilde{Q}_{N}\right]^{\top}$, and reference input vector $\widetilde{Q}_{\text {ref }}=$ $\left[\widetilde{Q}_{1, \text { ref }}, \ldots, \widetilde{Q}_{\mathrm{N}, \mathrm{ref}}\right]^{\top}$, where $(\cdot)^{\top}$ denotes the matrix transpose. After evaluation at the steady-state voltages $V_{j \mathrm{o}}=V_{\mathrm{g}} / M$ and 


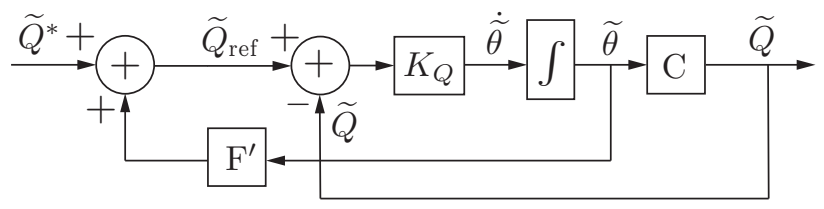

Fig. 4. Modified $Q$ control loop using state feedback.

angles $\theta_{j g_{0}}=\theta_{\mathrm{o}}$ for all $j \in\{1, \ldots, N\}$, (14) is written in matrix form $\widetilde{Q}=C \widetilde{\theta}$, where

$C=\frac{V_{\mathrm{g}}^{2}}{Z_{f} M^{2}}\left[\begin{array}{ccc}N-1-M \cos \theta_{\mathrm{o}} & \cdots & -1 \\ \vdots & \ddots & \vdots \\ -1 & \cdots & N-1-M \cos \theta_{\mathrm{o}}\end{array}\right]$

Small-signal perturbations in the control law in (11) give

$$
\dot{\tilde{\theta}}=\widetilde{\omega}=K_{Q}\left(\widetilde{Q}-\widetilde{Q}_{\mathrm{ref}}\right)
$$

Substitution of $\widetilde{Q}=C \widetilde{\theta}$ into (16) yields

$$
\dot{\tilde{\theta}}=A \widetilde{\theta}+B \widetilde{Q}_{\mathrm{ref}},
$$

where $A=K_{Q} C$, and $B=-K_{Q} I_{N} . I_{N}$ denotes the $N \times N$ identity matrix. The eigenvalues of $A$ are

$$
\begin{aligned}
& \lambda_{1}(A)=-K M \cos \theta_{\mathrm{o}}, \\
& \lambda_{2}(A)=\ldots=\lambda_{N}(A)=K\left(N-M \cos \theta_{\mathrm{o}}\right),
\end{aligned}
$$

where $K=K_{Q} V_{\mathrm{g}}^{2} /\left(Z_{\mathrm{f}} M^{2}\right)>0$. Evaluating the eigen values in (18)-(19) at the equilibrium points given in Section-II, we infer that $\theta_{\mathrm{o}} \approx 0$ and $M<0$ is an unstable equilibrium point. Moreover, $\theta_{\mathrm{o}} \approx 0$ and $M>0$ is a stable equilibrium point if and only if $M \cos \theta_{\mathrm{o}}>N$ or $M>N$. This from (3), requires that in steady state

$$
P_{j}=P_{\mathrm{o}}=\frac{V_{\mathrm{g}}^{2}}{M^{2} Z_{\mathrm{f}}}(N-M)<0
$$

and hence the inverter units must absorb active power. To bypass this restrictive condition and enable positive power transfer, next we modify the eigenvalues of $A$ via pole placement methods.

1) Stabilization via State Feedback-Based Pole Placement: Next, we modify the reactive power controller as depicted in Fig. 4. The reference input to the system is modified as $\widetilde{Q}_{\text {ref }}=$ $\widetilde{Q}^{\star}+F^{\prime} \widetilde{\theta}$, where $F^{\prime}$ is the state feedback matrix. Inserting the updated controller into (17) gives

$$
\dot{\tilde{\theta}}=(A+F) \widetilde{\theta}+B \widetilde{Q}^{\star},
$$

where $F=B F^{\prime}$. Since, the eigenvalues of $A$ depend on the diagonal elements, we choose $F$ as the diagonal matrix below:

$$
F=\left[\begin{array}{ccc}
-K m & \cdots & 0 \\
\vdots & \ddots & \vdots \\
0 & \cdots & -K m
\end{array}\right]
$$

where $m$ is a positive real parameter chosen such that the eigenvalues of the modified system matrix, $A+F$, remain negative despite variations in $M$. Note that the diagonal structure of $F$ preserves the decentralized nature of the envisioned system. The eigenvalues of $A+F$ are now,

$$
\begin{aligned}
& \lambda_{1}(A+F)=-K\left(m+M \cos \theta_{\mathrm{o}}\right), \\
& \lambda_{2}(A+F)=\cdots=\lambda_{N}(A+F)=K\left(N-m-M \cos \theta_{\mathrm{o}}\right)
\end{aligned}
$$

To ensure $\theta_{\mathrm{o}} \approx 0$ is a stable equilibrium point, the criteria $M+m>N$ must be satisfied. From (10), $M$ is minimized when the power delivered by all inverters is maximized; therefore, we must choose $m$ such that $M_{\min }+m>N$ is satisfied. This allows for control of active power delivery over the desired range. In every inverter, $Q_{j}^{\star}$ is adjusted to offset the effect of the state feedback and bring $Q_{j \text {,ref }}$ to a sufficiently small value to maintain a near-unity power factor operation. The integral action in the control loop maintains $Q_{j} \approx Q_{j, \text { ref }}$. This analysis also proves that the steady state where the angles of all inverters are synchronized among themselves and to the grid is stable, and there is no circulating reactive power among the inverters owing to $\theta_{j k}=0 \forall j, k \in\{1, \ldots, N\}$.

\section{B. Stability Analysis \& Design of P-Control Loop}

The active power control loop is designed to operate at a much faster rate than the reactive power loop. This ensures that the change in the voltage angles is much slower and that synchronism among the inverter units is maintained even when the units are undergoing transients in the processed active power. In effect, the reactive power control loop is invisible from the active power controller vantage point. Also, while in operation, the reactive power control loop is turned on first so that the units achieve the angle synchronization before the active power control loop is operated. Therefore, for the analysis below we assume $\theta_{j k} \approx 0$. From (1) we get

$$
P_{j}=\sum_{k=1}^{N} \frac{V_{j} V_{k}}{Z_{\mathrm{f}}}-\frac{V_{j} V_{\mathrm{g}}}{Z_{\mathrm{f}}} \cos \left(\theta_{j \mathrm{~g}}\right) .
$$

Perturbations in $V_{j}, V_{k}$ and $\theta_{j}$ around their steady-state values gives

$$
\begin{aligned}
\widetilde{P}_{j} \approx & \frac{1}{Z_{\mathrm{f}}}\left(\sum_{k=1}^{N} V_{k \mathrm{o}}+V_{j o}-V_{\mathrm{g}} \cos \theta_{j \mathrm{go}}\right) \widetilde{v}_{j}+\frac{V_{j \mathrm{o}}}{Z_{\mathrm{f}}} \sum_{k=1, \neq j}^{N} \widetilde{v}_{k} \\
& +\frac{V_{j \mathrm{o}} V_{\mathrm{g}} \sin \theta_{j \mathrm{go}}}{Z_{\mathrm{f}}} \widetilde{\theta}_{j} .
\end{aligned}
$$

Evaluating (26) at the steady-state voltages $V_{j \mathrm{o}}=V_{\mathrm{g}} / M$ and angles $\theta_{j \mathrm{go}}=\theta_{\mathrm{o}}$ for $j \in\{1, \ldots, N\}$ while considering small values of reactive power flow so that $\sin \theta_{j \text { go }} \approx 0$, we obtain

$$
\widetilde{P}_{j} \approx \frac{V_{\mathrm{g}}}{M Z_{\mathrm{f}}}\left((N+1)-M \cos \theta_{\mathrm{o}}\right) \widetilde{v}_{j}+\frac{V_{\mathrm{g}}}{M Z_{\mathrm{f}}} \sum_{k=1, \neq j}^{N} \widetilde{v}_{k} .
$$



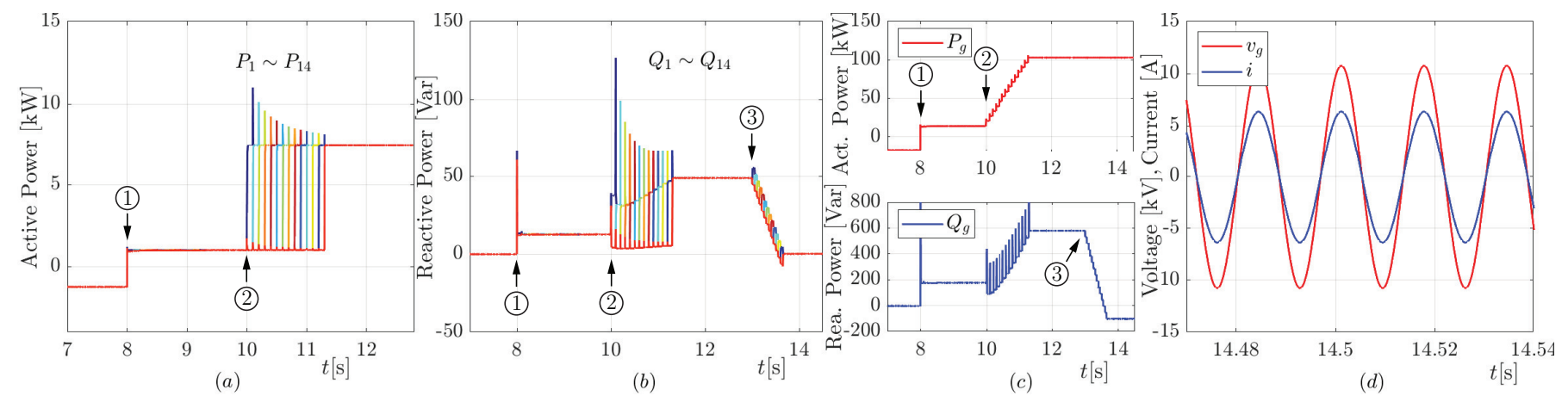

Fig. 5. Case study with state-feedback for stabilization. (a) Active power $P_{1}$ to $P_{14}$, (b) Reactive power $Q_{1}$ to $Q_{14}$, (c) Active and reactive power delivered to grid, $P_{\mathrm{g}} \& Q_{\mathrm{g}}$, respectively, and (d) Grid voltage $v_{\mathrm{g}}$ and current $i$. Events (1), (2), and (3) correspond to the active power control loop turn-on, active power command step changes, and reactive power step changes, respectively.
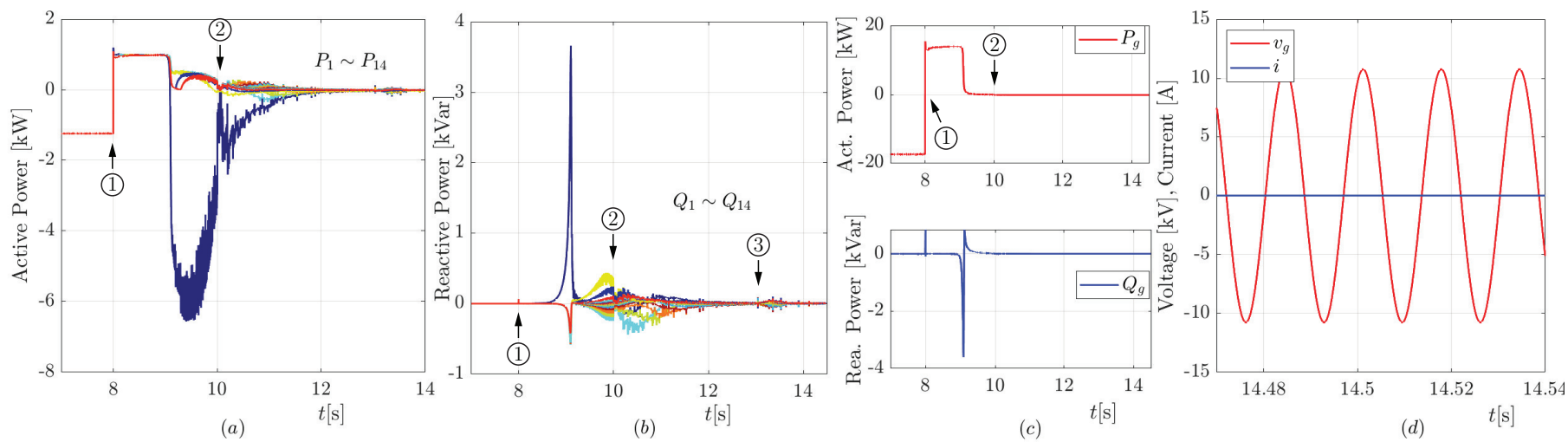

Fig. 6. Unstable system where $Q$-loop state feedback is neglected. Variables in each plot and events are identical to that in Fig. 5.

After we define the state vector $\widetilde{v}=\left[\widetilde{v}_{1}, \ldots, \widetilde{v}_{N}\right]^{\top}$, and output vector $\widetilde{P}=\left[\widetilde{P}_{1}, \ldots, \widetilde{P}_{N}\right]^{\top},(27)$ is written in matrix form $\widetilde{P}=G \widetilde{v}$, where

$G=\frac{V_{\mathrm{g}}}{M Z_{f}}\left[\begin{array}{ccc}N+1-M \cos \theta_{\mathrm{o}} & \cdots & 1 \\ \vdots & \ddots & \vdots \\ 1 & \cdots & N+1-M \cos \theta_{\mathrm{o}}\end{array}\right]$.

We rewrite (12) in the following small-signal form

$$
\dot{\widetilde{v}}=K_{P}\left(\widetilde{P}_{\text {ref }}-\widetilde{P}\right),
$$

where the active power reference vector is $\widetilde{P}_{\text {ref }}=$ $\left[\widetilde{P}_{1, \text { ref }}, \ldots, \widetilde{P}_{\mathrm{N}, \text { ref }}\right]^{\top}$. Substitution of $\widetilde{P}=G \widetilde{v}$ into (29) gives the voltage state equation

$$
\dot{\tilde{v}}=-K_{P} G \widetilde{v}+K_{P} \widetilde{P}_{\text {ref }} .
$$

The eigenvalues of the system matrix $-K_{P} G$ in this case are

$$
\begin{aligned}
& \lambda_{1}=-k^{\prime} N, \\
& \lambda_{2}=\ldots=\lambda_{N}=-k^{\prime}\left(N-M \cos \theta_{\mathrm{o}}\right),
\end{aligned}
$$

where $k^{\prime}=K_{P} V_{\mathrm{g}} /\left(M Z_{f}\right)$. By inspecting the eigen values in (31)-(32), we infer that the equilibrium defined by $\theta_{\mathrm{o}} \approx 0$ and $M<0$ is unstable. However, the equilibrium point near $\theta_{\mathrm{o}} \approx 0$ and $M>0$ is stable in the case when $M \cos \theta_{\mathrm{O}}<N$ or $M<N$. This implies that the $P$-control loop is stable under positive active power flow at the desired equilibrium point near $\theta_{\mathrm{o}} \approx 0$.

\section{Simulation Results}

The proposed control scheme was simulated for a system of $N=14 \mathrm{H}$-bridge inverters connected to a medium-voltage grid. The simulated system parameters are in Table I. The active power controller is designed to operate 20 times faster than the reactive power controller so that their operation are appropriately decoupled from each other. This is done by choosing $K_{P}=100$ and $K_{Q}=0.01$ such that the eigen values are appropriately scaled. Next, two cases are simulated. In Case 1, the inverters are controlled using the control law outlined in (11)-(12) with the proposed state feedback stabilization in the $Q$ control loop. Next, Case 2 illustrates the necessity of the state-feedback stabilization where the system is operated identically to Case 1 but without state feedback. In both cases, voltage synchronisation is first achieved via the $Q$ control loop with $Q_{j, \text { ref }}=0$ for all units. At $t=8 \mathrm{~s}$, the $P$-control loop is activated (denoted as event (1) with $P_{j, \text { ref }}=1 \mathrm{~kW} \forall j \in\{1, \ldots, N\}$. Starting at $t=10 \mathrm{~s}, P_{j, \text { ref }}$ is 
stepped up from $1 \mathrm{~kW}$ to $7.5 \mathrm{~kW}$ at $t=10+0.1(j-1) \mathrm{s}$ for the $j^{\text {th }}$ inverter (see event (2)).

TABLE I

SYSTEM AND CONTROLLER PARAMETERS.

\begin{tabular}{lllcl}
\hline \hline \multirow{2}{*}{ Symbol } & & Description & Value & Units \\
\cline { 1 - 3 } \cline { 1 - 1 }$V_{\mathrm{dc}}$ & & dc link voltage & 1000 & $\mathrm{~V}$ \\
$\omega_{\mathrm{o}}$ & & nominal grid frequency & $2 \pi 60$ & $\mathrm{rad} / \mathrm{s}$ \\
$V_{\mathrm{g}}$ & & line-neutral RMS grid voltage & 7.62 & $\mathrm{kV}$ \\
$R_{\mathrm{v}}$ & & virtual resistance per inverter & 2.5 & $\Omega$ \\
$P_{\mathrm{o}}$ & & rated active power per inverter & 7.5 & $\mathrm{~kW}$ \\
$K_{Q}$ & & Q controller gain & 0.01 & $\mathrm{rad} /(\mathrm{VAR} \mathrm{s})$ \\
$K_{P}$ & & P controller gain & 100 & $\mathrm{~V} / \mathrm{J}$ \\
$M_{\min }$ & & minimum value of $M$ & 13.21 & - \\
$m$ & state feedback gain & 3 & - \\
\hline \hline
\end{tabular}

Case 1 results are shown in Fig. 5. Due to state feedback, the system remains stable even after the active power control is turned on and both control loops are active. Fig. 5(a) and (b), respectively, show the active and reactive power tracking for all the 14 units. Starting from $t=13 \mathrm{~s}, Q_{j}^{*}$ is changed from 0 to $-50 \mathrm{VAR}$ at $t=13+0.1(j-1) \mathrm{s}$ for the $j^{\text {th }}$ inverter in order to drive the grid reactive power near zero (observe event (3). Fig. 5(c) shows the overall active and reactive power delivered to the grid. At $t=10 \mathrm{~s}$, it is evident that the active power delivered to the grid increases from $14 \mathrm{~kW}$ to $105 \mathrm{~kW}$ in small steps of $6.5 \mathrm{~kW}$, as the inverters increase their active power output one at a time. The net reactive power absorbed from the grid is made as low as $100 \mathrm{VAR}$, ensuring a near unity power factor. Fig. 5(d) shows the grid voltage and the grid current.

Case 2 waveforms are shown in Fig. 6. As shown in Fig. 6(b), the system goes unstable once the power control loop is activated despite the attainment of synchronization beforehand. Active power instability is highlighted in Fig. 6(a) after a change in commands.

\section{CONCLUSion}

In this paper, we proposed and validated a novel control method to achieve decentralized control of active and reactive power in a system of series-connected converters tied to the grid. The proposed controller processes only the locally sensed current signal at each inverter and synthesizes the output voltage of each inverter using two decoupled control loops for active and reactive power. The reactive power control loop generates the voltage angle and maintains synchronism among the series-connected inverters, whereas the active power control loop generates the voltage amplitude that governs the active power injected by each inverter. The design method and small-signal stability analysis of both the control loops are provided in the paper. The proposed approach was validated in a simulation of a system comprising 14 inverter units connected in series and delivering power to the grid.

\section{REFERENCES}

[1] A. Dell'Aquila, M. Liserre, V. G. Monopoli, and P. Rotondo, "Overview of pi-based solutions for the control of dc buses of a single-phase h-bridge multilevel active rectifier," IEEE Transactions on Industry Applications, vol. 44, no. 3, pp. 857-866, 2008.

[2] T. Zhao, G. Wang, S. Bhattacharya, and A. Q. Huang, "Voltage and power balance control for a cascaded h-bridge converter-based solidstate transformer," IEEE Transactions on Power Electronics, vol. 28, no. 4, pp. 1523-1532, 2012.

[3] S. Kouro, B. Wu, Á. Moya, E. Villanueva, P. Correa, and J. Rodríguez, "Control of a cascaded h-bridge multilevel converter for grid connection of photovoltaic systems," in 2009 35th Annual Conference of IEEE Industrial Electronics, pp. 3976-3982, IEEE, 2009.

[4] E. Villanueva, P. Correa, J. Rodríguez, and M. Pacas, "Control of a single-phase cascaded h-bridge multilevel inverter for grid-connected photovoltaic systems," IEEE Transactions on industrial electronics, vol. 56, no. 11, pp. 4399-4406, 2009.

[5] A. Marzoughi, R. Burgos, D. Boroyevich, and Y. Xue, "Investigation and comparison of cascaded h-bridge and modular multilevel converter topologies for medium-voltage drive application," in IECON 2014-40th Annual Conference of the IEEE Industrial Electronics Society, pp. 15621568, IEEE, 2014.

[6] P. K. Achanta, B. B. Johnson, G.-S. Seo, and D. Maksimovic, "A multilevel DC to three-phase AC architecture for photovoltaic power plants," IEEE Transactions on Energy Conversion, vol. 34, no. 1, pp. 181-190, 2018.

[7] L. Zhang, K. Sun, Y. W. Li, X. Lu, and J. Zhao, "A distributed power control of series-connected module-integrated inverters for PV grid-tied applications," IEEE Transactions on Power Electronics, vol. 33, no. 9, pp. 7698-7707, 2017.

[8] P. K. Achanta, D. Maksimovic, and M. Ilic, "Decentralized control of series stacked bidirectional DC-AC modules," in Applied Power Electronics Conference and Exposition, pp. 1008-1013, IEEE, 2018.

[9] D. Evans and R. Cox, "Powerline communications strategy enabling fully decentralized control of AC-stacked PV inverters," in Energy Conversion Congress and Exposition, pp. 2277-2284, IEEE, 2017.

[10] J. He, Y. Li, C. Wang, Y. Pan, C. Zhang, and X. Xing, "Hybrid microgrid with parallel-and series-connected microconverters," IEEE Transactions on Power Electronics, vol. 33, no. 6, pp. 4817-4831, 2017.

[11] D. M. Scholten, N. Ertugrul, and W. Soong, "Analysis and control of decentralized pv cascaded multilevel modular integrated converters," in 2016 IEEE Energy Conversion Congress and Exposition (ECCE), pp. 19, IEEE, 2016.

[12] H. Jafarian, N. Kim, B. Parkhideh, and J. Enslin, "Analysis of smart inverter functions of decentralized grid-connected ac-stacked pv inverter architecture," in 2017 IEEE Applied Power Electronics Conference and Exposition (APEC), pp. 3594-3600, IEEE, 2017.

[13] J. He, Y. Li, B. Liang, and C. Wang, "Inverse power factor droop control for decentralized power sharing in series-connected-microconvertersbased islanding microgrids," IEEE Transactions on Industrial Electronics, vol. 64, no. 9, pp. 7444-7454, 2017.

[14] P. Achanta, M. Sinha, B. Johnson, S. Dhople, and D. Maksimovic, "Selfsynchronizing series-connected inverters," in Workshop on Control and Modeling for Power Electronics, pp. 1-6, IEEE, 2018.

[15] X. Hou, Y. Sun, X. Zhang, G. Zhang, J. Lu, and F. Blaabjerg, "A self-synchronized decentralized control for series-connected H-bridge rectifiers," IEEE Transactions on Power Electronics, vol. 34, no. 8, pp. 7136-7142, 2019.

[16] X. Hou, Y. Sun, H. Han, Z. Liu, W. Yuan, and M. Su, "A fully decentralized control of grid-connected cascaded inverters," IEEE Transactions on Sustainable Energy, vol. 10, no. 1, pp. 315-317, 2018.

[17] W. Yao, M. Chen, J. Matas, J. M. Guerrero, and Z.-M. Qian, "Design and analysis of the droop control method for parallel inverters considering the impact of the complex impedance on the power sharing," IEEE Transactions on Industrial Electronics, vol. 58, no. 2, pp. 576-588, 2010. 